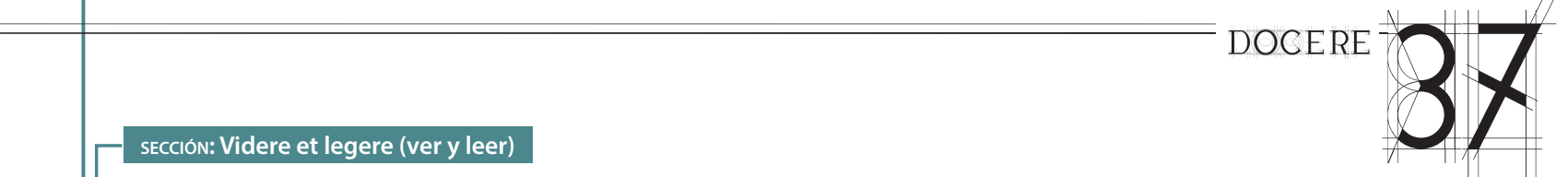

\title{
¿Qué son y cómo implementar los métodos de enseñanza en el aula?
}



Díaz, Mario de Miguel y cols. (2005). Modalidades de enseñanza centradas en el desarrollo de competencias. Orientaciones para promover el cambio metodológico en el Espacio Europeo de Educación Superior. España: Universidad de Oviedo ${ }^{1}$

E ste libro fue desarrollado por Mario de Miguel Díaz, junto con un equipo de investigación conformado por profesionistas de diversas universidades de España: la Universidad de Valencia, del País Vasco, de Oviedo y de Sevilla. Entre los principales colaboradores se encuentran: Ignacio Javier Alfaro Rocher, Pedro Apodaca Urquijo, José Miguel Arias Blanco, Eduardo García Jiménez, Clemente Lobato Fraile y Alfredo Pérez Boullosa.

Los autores describen un modelo educativo en el cual las competencias adquiridas por el estudiante son un elemento esencial en el proceso de planificación, ya que el actuar del docente consistirá en organizar los elementos que propicien el logro de estas competencias. Por tanto, el profesor habrá de considerar las modalidades organizativas o escenarios

1 Versión digital disponible en: http://bit.ly/1ricsKa. 
de aprendizaje, los métodos de trabajo por implementar y el sistema de evaluación que se llevará a cabo en el proceso educativo.

En este sentido, en primer lugar el lector se adentra a una aproximación del concepto de competencia, su tipología, y los elementos necesarios para su desarrollo.

Por otro lado, en el texto se presenta una clasificación y descripción de algunas modalidades organizativas o escenarios de aprendizaje, como las clases teóricas, clases prácticas, tutorías, estudio y trabajo en grupo, entre otras. A través de estas descripciones, el docente podrá identificar cómo planificar e implementar cada modalidad.

Posteriormente, los autores destacan la importancia de los métodos de enseñanza empleados por el docente. En este sentido, dan a conocer su concepto, de acuerdo a sus funciones y características principales, y exponen algunos de los que se han utilizado con mayor frecuencia en el contexto universitario.

Dicha exposición o propuesta es uno de los elementos más enriquecedores de la obra, ya que se presentan diferentes métodos, como el expositivo, estudio de casos, resolución de ejercicios y problemas, aprendizaje orientado a proyectos, aprendizaje colaborativo, contrato de aprendizaje, entre otros. Asimismo, se describe la finalidad de cada uno, sus características, las tareas y competencias del profesor y del estudiante, lo que habrá de tomarse en cuenta para su planificación, recursos necesarios, procedimientos de evaluación a utilizar, beneficios y limitantes, y bibliografía relacionada tanto en formato impreso como en electrónico.

Finalmente, los autores ofrecen un panorama respecto a los sistemas de evaluación y sus implicaciones dentro del contexto de las competencias, algunas de sus estrategias, procedimientos y técnicas evaluativas, y la relación que éstas guardan con los métodos de enseñanza referidos.

Lo expresado anteriormente es de suma importancia para que el docente lleve a cabo su actuar diario de acuerdo con las necesidades de aprendizaje de los estudiantes. Esta publicación proporciona herramientas de una manera puntual, guiando paso a paso a los profesores interesados en la aplicación de metodologías de enseñanza y aprendizaje efectivas, de acuerdo a lo que exige la educación en la actualidad.

Mario de Miguel Díaz, autor de la obra y catedrático de la Facultad de Ciencias de la Educación de la Universidad de Oviedo, es reconocido por diversas publicaciones relacionadas con metodologías de enseñanza y aprendizaje, evaluación de programas, desarrollo profesional del profesorado, formación profesional para el empleo, entre otros. En junio del año 2014 recibió un reconocimiento por su compromiso con la educación pública. 